\title{
18F-choline positron emission tomography/computed tomography guided laparoscopic salvage lymph node dissection in patients after radical prostatectomy
}

\author{
Markiian Kubis, Krystian Kaczmarek, Artur Lemiński, Marcin Słojewski \\ Department of Urology and Urological Oncology, Pomeranian Medical University, Szczecin, Poland
}

Videosurgery Miniinv 2021; 16 (2): 403-408 DOI: https://doi.org/10.5114/wiitm.2020.100738

\begin{abstract}
Introduction: Salvage lymph node dissection ( $L N D$ ) using novel imaging methods is an interesting alternative to treat lymph node (LN) metastasis after radical prostatectomy (RP); however, recommendations for using sLND as such are still being developed.

Aim: To assess the clinical outcomes of prostate cancer (PCa) after fluorine-18-choline (18F-choline) positron emission tomography/computed tomography (PET/CT) guided sLND.

Material and methods: Ten patients who had undergone SLND under 18F-choline PET/CT guidance (positive nodes: median 1, range 1-3) and had biochemical recurrence or persistence of prostate-specific antigen (PSA: median PSA $2.05 \mathrm{ng} / \mathrm{ml}$, range: $0.8-8.4$ ) after $R P$ were enrolled in this retrospective study. Complete biochemical response (CBCR) after salvage surgery was defined as PSA $<0.2 \mathrm{ng} / \mathrm{ml}$.

Results: The median follow-up time after salvage surgery was 33 months. The median PSA level 6 weeks after sLND and at the end of follow-up was 0.93 and $2.95 \mathrm{ng} / \mathrm{ml}$, respectively. At 6 weeks after targeted sLND only 1 patient had $C B C R$, whereas a PSA decrease was noted in 7 patients. No patient had CBCR at the end of follow-up.

Conclusions: Laparoscopic SLND in cases of LN metastatic PCa after RP is a feasible option with low morbidity. However, an initial $C B C R$ occurs in a negligible proportion of patients, and a long-term response is unlikely to be achieved.
\end{abstract}

Key words: prostate cancer, radical prostatectomy, choline, salvage lymph node dissection, fluorine-18.

\section{Introduction}

Radical prostatectomy (RP) remains one of the most common treatment methods for prostate cancer (PCa) without metastasis [1-3]. As a result, within the last few years in Poland, the total number of procedures conducted and the number of the centres reporting the use of laparoscopic or robotic RPs have increased substantially [4]. This surgery is performed with curative intent, although between $27 \%$ and $53 \%$ of all patients develop biochemical recurrence $(B C R)$ [5]. Subsequently, the most com- mon choice of treatment is salvage radiotherapy (SRT), which is indicated when prostate-specific antigen (PSA) is below $0.5 \mathrm{ng} / \mathrm{ml}$ [6]. SRT is usually performed without additional imaging. However, novel imaging methods allow for the identification of tumour cell sites and further targeted management, such as salvage lymph node dissection (sLND) [7]. It is important to note that intra- and postoperative complications and the effectiveness of sLND are unknown, which limits the use of this approach [8]. Nevertheless, a reduction in operation time, which consequently leads to a decreased number of post-

\section{Address for correspondence}

Krystian Kaczmarek MD, Department of Urology and Urological Oncology, Pomeranian Medical University, Szczecin, Poland,

phone: +48 662552841, e-mail: k.kaczmarek.md@gmail.com 
operative complications (such as lymphocele), may be achieved by performing targeted lymphadenectomy.

\section{Aim}

The aim of this study was to analyse the outcomes and evaluate the benefits of targeted SLND after RP.

\section{Material and methods}

Patients who underwent laparoscopic RP with pelvic lymph node (LN) dissection in the urology department between January 2016 and December 2017 were included in the study. Retrospective data analysis identified patients with PSA recurrence or persistence after surgery. Further, patients were selected according to the results of ${ }^{18} \mathrm{~F}$-choline positron emission tomography/computed tomography (PET/ $\mathrm{CT}$ ) scans. Patients had to have at least one PET-positive LN and no evidence of a local recurrence or bone metastases in conventional diagnostic imaging (ultrasound and/or computed tomography and/or magnetic resonance and/or bone scintigraphy) in order to be included. Finally, patients who underwent targeted SLND were identified, and those treated with other therapeutic options before sLND were not excluded. The final sample included 10 patients. Mean age was 66.8 years (standard deviation 5.25 ).

PSA level was tested before RP, the day of ${ }^{18} \mathrm{~F}$-choline PET/CT examination, 6 weeks after SLND and $3,6,9$, and 12 months after sLND; and biannually thereafter. PET/CT scans were not routinely repeated after salvage surgery. The follow-up was ended when a second salvage therapy was initiated. Complete biochemical response (CBCR) after sLND was defined as PSA $<0.2 \mathrm{ng} / \mathrm{ml}$. PSA decrease after surgery without reaching $C B C R$ was defined as PSA-decrease-no response.

All salvage procedures were first theoretically planned in a consensus conference with at least 2 urological surgeons. Only PET/CT-positive and nearby LNs were resected. The nearby LNs were defined as LNs located in the same anatomical area as PET/CT-positive LN. For example, if the external iliac lymph node was positive on PET/CT, the residual LN overlapping the external iliac vessel was removed.

During surgery, the surgeons had access to the imaging material. Thus, if adjustments were needed, their approaches were based on the imaging infor- mation. All salvage procedures were performed by a transperitoneal laparoscopic approach.

The resected LNs were labelled according to location, sectioned at 3-mm intervals, formalin fixed and submitted for paraffin embedding. Micro-slices were placed on glass slides and stained with haematoxylin and eosin. Immunohistochemistry was performed if needed.

All ${ }^{18} \mathrm{~F}$-choline PET/CT scans were performed at one external centre by experts using integrated PET/ $\mathrm{CT}$ systems. Experienced radiologists and nuclear medicine specialists assessed the images and located the sites of pathological choline uptake. The diagnosis of tumour-positive LNs on PET/CT images was based on visual evidence of increased focal uptake on a choline PET scan, where the location corresponded to LNs on CT images.

Since only PET/CT-positive and adjacent LNs were removed and no extended lymphadenectomy was performed, it was not possible to calculate sensitivity (SN), specificity (SP) or negative predictive value (NPV). Positive predictive value (PPV) was calculated according to its definition (true-positive/(true-positive + false-positives)).

\section{Results}

Table I shows a summary of patient characteristics. The overall median (range) pre-sLND PSA value was 2.05 (0.8-8.4) ng/ml. Overall, 114 nodes in 10 patients were excised. The median number of positive nodes was 1 (range: 1-6). Before SLND, 4 patients received salvage radiotherapy (SRT). The PPV of ${ }^{18} \mathrm{~F}$-choline PET/CT in detecting LN metastases was $90 \%$. Mean operation time and mean hospital stay were $97.5 \mathrm{~min}$ and 3 days, respectively. No patient had intra-operative complications, or required an open conversion or any blood transfusions. At follow-up, no lymphocele was reported.

Median (range) follow-up time after salvage surgery was 22 (10-33) months. The median PSA level 6 weeks after sLND and at the end of follow-up was 0.93 and $2.95 \mathrm{ng} / \mathrm{ml}$, respectively. When assessing the dynamics of PSA level within 6 weeks after targeted SLND only 1 patient had CBCR (PSA level $<0.2 \mathrm{ng} / \mathrm{ml}$ ), but PSA-decrease-no response was noted in 7 patients. The patient with $C B C R$ developed local metastasis at follow-up and finally received chemotherapy with docetaxel. One patient with initial PSA-decrease-no response received androgen deprivation therapy (ADT) due to PSA rising at the 
Table I. Baseline characteristics of patients

\begin{tabular}{|c|c|c|c|c|c|c|c|c|c|}
\hline No. & $\begin{array}{c}\text { Age } \\
\text { [years] }\end{array}$ & $\begin{array}{c}\text { PSA } \\
1\end{array}$ & TNM stage & $\begin{array}{l}\text { Gleason } \\
\text { score }\end{array}$ & $\begin{array}{c}\text { PSA } \\
2\end{array}$ & $\begin{array}{l}\text { Localization of positive } \\
\text { uptake }\end{array}$ & $\begin{array}{l}\text { Histology } \\
\text { results }\end{array}$ & $\begin{array}{c}\text { PSA } \\
3\end{array}$ & $\begin{array}{c}\text { PSA } \\
4\end{array}$ \\
\hline 1 & 70 & 10.5 & pT2b pNx cMO RO & $3+3$ & 8.4 & Left common iliac vessels & Positive & 2.1 & 2.9 \\
\hline 2 & 61 & 7.5 & pT2c pNO cMO R1 & $4+4$ & 2.1 & Right external iliac vessels & Positive & 0.9 & 1.6 \\
\hline 3 & 76 & 3.7 & pT2c pNO cMO RO & $4+4$ & 3.3 & Left external iliac vessels & Positive & 1.5 & 2.0 \\
\hline 4 & 67 & 9.1 & pT2c pNO cMO R1 & $4+3$ & 7.54 & Left common iliac vessels & Positive & 0.3 & 11 \\
\hline 5 & 62 & 7.8 & pT2b pNO cMO RO & $4+3$ & 1.3 & Right obturator fossa & Positive & 0.8 & 2.0 \\
\hline 6 & 72 & 3.4 & pT3a pN1 cM0 R0 & $4+4$ & 2.3 & One paracaval lymph node & Positive & 0.09 & 10.8 \\
\hline 7 & 70 & 12.0 & pT2c pNO cMO RO & $4+3$ & 1.3 & $\begin{array}{l}\text { Right obturator fossa and } \\
\text { right parailiac Ins }\end{array}$ & Negative & 0.7 & 5.1 \\
\hline 8 & 67 & 7.8 & pT3a pNO cMO RO & $3+4$ & 2.0 & Right common iliac vessels & Positive & 1.4 & 1.9 \\
\hline 9 & 63 & 17.0 & pT3b pNO cMO RO & $3+4$ & 8.0 & Right iliac external vessels & Positive & 9.1 & 13.0 \\
\hline 10 & 60 & 10 & pT2c pNO cM0 R1 & $3+3$ & 0.8 & $\begin{array}{c}\text { Left iliac external and internal } \\
\text { vessels }\end{array}$ & Positive & 0.95 & 3.0 \\
\hline
\end{tabular}

PSA 1 - PSA at diagnosis, PSA 2-PSA at PET/CT, PSA 3-PSA 6 weeks after SLND, PSA 4-PSA at the end of follow-up.

follow-up. Remaining patients with PSA-decrease no response were managed by close monitoring.

Increase in PSA level was noted in 2 patients at 6 weeks after sLND. One of them was treated with ADT.

\section{Discussion}

The role of different PET/CT tracers in detecting recurrence after radical therapy of PCa has been assessed in numerous studies. Prostate-specific membrane antigen (PSMA) PET/CT is considered to be better than conventional and PET imaging with other tracers, including choline PET/CT, especially in the context of BCR [8]. Hence, the European Association of Urology guidelines recommend PSMA PET/CT imaging in BCR when low PSA levels occur, particularly if the PSA level is $1 \mathrm{ng} / \mathrm{ml}$ or more after RP [5]. However, PSMA PET/CT imaging is not readily available in Poland. Therefore, choline PET/CT is commonly performed when BCR is detected. The pooled detection rate of choline PET/CT, SN and SP for BCR for all sites of recurrence is $62 \%, 89 \%$ and $89 \%$, respectively [6]. In LN metastasis, a lesion-based analysis showed that ${ }^{11} \mathrm{C}$-choline PET/CT SP, SP, PPV, NPV, and accuracy were $64 \%, 90 \%, 86 \%, 72 \%$, and $77 \%$, respectively [9]. The SN mainly depends on PSA level and may range between 38 and 98\% [6]. The low NPV seems to depend on the restricted capability of ${ }^{11} \mathrm{C}$-choline PET/CT to detect microscopic lesions. However, the high PPV, even with low PSA values, provides a basis for further treatment decisions [9].
The surgical management of LN metastases after RP has been the topic of several retrospective analyses. However, no consensus has been reached about the extent of $\mathrm{LN}$ dissection and the templates that need to be dissected during salvage procedures. Most studies reported results for an anatomically defined, extended SLND that included the obturator fossa; external, internal, and common iliac artery regions; and proximal removal of all lymph nodes along the common iliac vessels to the aortic bifurcation $[10,11]$. In addition, in some studies the dissection was extended to the retroperitoneum in the case of positive spots above the aortic bifurcation [12-15]. Jilg et al. presented results of open extended sLND after radical radiotherapy or prostatectomy. $\mathrm{PCa}$ recurrence in $\mathrm{LN}$ was confirmed by ${ }^{11} \mathrm{C} /{ }^{18} \mathrm{~F} \mathrm{PET} /$ CT [16]. During the histopathological evaluation of the removed LNs, PCa was verified in all patients. Jilg et al. emphasised the high diagnostic accuracy of choline PET/CT for pelvic and retroperitoneal LNs regions, and concluded that if there is one choline-positive $L N$ in an iliac subregion, then all pelvic subregions on that side should be resected to sufficiently eradicate micro-metastatic lymphatic spread [16]. Deconinck et al. also assessed the diagnostic value of ${ }^{11} \mathrm{C}$-choline PET/CT scan to detect $L N$ metastases in patients with $B C R$ after radically treated $\mathrm{PCa}$ [17]. Contrary to the findings of Jilg et al., Deconinck et al. found that the overall detection using choline $\mathrm{PET} / \mathrm{CT}$ rate was relatively low with a moderate PPV 
for metastatic LNs in patients with BCR. Additionally, a high rate of false-positive nodes, which were mainly located in the external iliac regions, was observed. These findings seem to discourage the planning process of highly selective metastatic ablation, which focused only on positive spots of choline PET/ CT scans [17].

Evidence for targeted sLNDs is limited. Winter et al. presented results of guided SLND in patients with PSA failure and single LN recurrence after RP [18]. Following the salvage surgery, 10 of 11 patients with histologically confirmed LN metastases showed a PSA response. In 5 patients, the PSA value decreased below $0.2 \mathrm{ng} / \mathrm{ml}$. PSA was examined without ADT, and the median follow-up was 72 months. According to Winter et al., this approach at least offers a therapeutic benefit in selected cases with minimal lymphatic dissemination [18]. In contrast to the Winter et al. study, the current study showed SLND with a less promising approach. Despite the fact that our study had a shorter median follow-up time (compared to Winter et al.), and 2 patients received ADT, no patient achieved long-lasting, complete PSA remission.

Siriwardana et al. compared different templates of $L N$ resection [19]. After reviewing primary, adjuvant and salvage treatment history, preoperative $\mathrm{PET} / \mathrm{CT}$ imaging, and intra-operative anatomical findings, patients were selected to undergo a targeted sLND limited to the PET/CT detected lesions and any surrounding lymph nodes and a unilateral or bilateral extended SLND. The Siriwardana et al. study revealed that the only significant predictor for treatment response was bilateral template dissection, with an odds ratio of 28.5 ( $95 \% \mathrm{Cl}: 4.17-584.92)$ for PSA level below $0.2 \mathrm{ng} / \mathrm{ml}$ [19].

Conflicting results for choline PET/CT based targeted sLND emphasise that this strategy remains controversial. Choline PET/CT cannot detect LNs smaller than $8 \mathrm{~mm}$ [20]. Of course, the positive predictive value is high. However, choline PET/ CT can only identify patients with large nodes and advanced disease, whereas patients with smaller nodes are negative. In positive patients, when excising the large positive nodes only, a number of choline PET/CT negative metastatic nodes may remain. This explains our poor results. In contrast, patients with small metastatic nodes only and therefore more suited for SLND cannot be selected by means of choline $\mathrm{PET} / \mathrm{CT}$. Therefore, new PET/CT tracers were investigated in this issue. Recently, Maurer et al. assessed the feasibility of 99m-technetium-based, PSMA-radioguided surgery in patients recurring after local treatment and selected by an initial ${ }^{68}$ gallium-labelled PSMA $\left({ }^{68} \mathrm{Ga}\right.$-PSMA) ligand PET/CT. Oncological outcomes were overall at least as good as those previously reported in bilateral extended SLND series [10]. This may suggest that that the improvements of preoperative imaging could outline more precise removal fields. Nonetheless, when ${ }^{68} \mathrm{Ga}$-PSMA PET/ CT was used, bilateral extended SLND was more likely to provide $C B C R$, suggesting that identification of small and/or atypically localised lesions during salvage surgery procedures is challenging [19]. Hence, the accuracy of PSMA-PET remains insufficient to justify resection of only PSMA-positive fields, but favours complete bilateral SLND.

Today, minimally invasive approaches are the most widely used for SLND. Kolontarev et al. presented results of 10 patients with only identified LN metastases of PCa. All patients underwent robotic extended sLND. Overall median PSA decreased by $31.4 \%$, and no patient reached a PSA of zero postoperatively. One patient had Clavien-Dindo grade II complications. Kolontarev et al. concluded that a robotic approach is safe and allows for the majority of patients to postpone ADT, which theoretically decreases treatment costs [21]. A laparoscopic approach was assessed by Schilling et al., and all 10 patients initially presented PSA regress after SLND. At follow-up, 2 patients were being closely monitored, 2 had received radiotherapy of the prostate fossa, and 2 had received chemotherapy with docetaxel. In addition, 4 patients were treated by ADT in the Schilling et al. study. No blood transfusions, conversion to open surgery, or perioperative complications were reported [22].

The current study provides valuable information about the clinical course after SLND in PCa patients. Nevertheless, this study had some limitations. First, there was insufficient information on PSA kinetics after RP available for the selected patients. PSA level, PSA doubling time and PSA velocity are correlated with PET/CT detection rates and should be taken into account when considering the use of choline PET/CT [23]. Also, additional ${ }^{18}$ F-choline PET/CT scans were not carried out as a standard procedure in all $10 \mathrm{pa}-$ tients after SLND. This means that some true-positive LNs might not have been removed, which can be a plausible explanation for failure of surgery in patient no. 7, who presented no histologically proven LN metastasis in the SLND specimen. Furthermore, 
the study was limited by the small sample size and the non-homogeneous distribution of the study cohort. Some patients were previously treated by ADT or radiotherapy. Therefore, it is unclear if these results will be reproducible for a homogeneous population without previous therapy. Patients with previous hormonal therapy might have had suppressed PSA levels, which might not correlate with tumour size or metabolism [24].

\section{Conclusions}

Choline PET/CT SLND is associated with an initial CBCR in a negligible proportion of patients. Moreover, even if the initial response is achieved, a prolonged response without additional treatment is unlikely. This supports the hypothesis that targeted choline PET/CT sLND removes only the start of more micro-metastases that are likely to present at this stage. Therefore, based on the current study and conflicting results of previous studies, we cannot recommend this approach as proper treatment of LN metastasis. However, this study is not suited to draw conclusions on the effectiveness of salvage lymph node dissection with other new PET/CT tracers.

\section{Conflict of interest}

The authors declare no conflict of interest.

\section{References}

1. Benoit RM, Naslund MJ, Cohen JK. Complications after radical retropubic prostatectomy in the medicare population. Urology 2000; 56: 116-20.

2. Jarzemski P, Listopadzki S, Słupski P, et al. Laparoscopic radical prostatectomy and extended pelvic lymph node dissection: a combined technique. Videosurgery Miniinv 2020; 15: 192-8.

3. Kozikowski M, Zagożdżon B, Gola M, Dobruch J. Prostate Imaging Reporting and Data System in prostate cancer staging and planning for radical prostatectomy. Videosurgery Miniinv 2019; 14: 262-70

4. Interpelacja nr 6090 - tekst odpowiedzi [Internet]. [cited 2020 Jan 17]. Available from: http://www.sejm.gov.pl/sejm8.nsf/InterpelacjaTresc.xsp?key=7B1C9152

5. Lam TBL, Maclennan S, Willemse PPM, et al. EAU-EANM-ES TRO-ESUR-SIOG Prostate Cancer Guideline Panel Consensus Statements for Deferred Treatment with Curative Intent for Localised Prostate Cancer from an International Collaborative Study (DETECTIVE Study). Eur Urol 2019; 76: 790-813.

6. Tam HH, Barwick TD, Khan SR, et al. Clinical PET imaging in prostate cancer - erratum. RadioGraphics 2017; 37: 2208.

7. Fossati N, Suardi N, Gandaglia G, et al. Identifying the optimal candidate for salvage lymph node dissection for nodal recur- rence of prostate cancer: results from a large, multi-institutional analysis. Eur Urol 2019; 75: 176-83.

8. Witkowska-Patena E, Mazurek A, Dziuk M. 68Ga-PSMA PET/CT imaging in recurrent prostate cancer: Where are we now? Cent Eur J Urol 2017; 70: 37-43.

9. Scattoni V, Picchio M, Suardi N, et al. Detection of lymph-node metastases with integrated [11C]choline PET/CT in patients with psa failure after radical retropubic prostatectomy: results confirmed by open pelvic-retroperitoneal lymphadenectomy. Eur Urol 2007; 52: 423-9.

10. Maurer T, Robu S, Schottelius M, et al. 99m Technetium-based prostate-specific membrane antigen-radioguided surgery in recurrent prostate cancer. Eur Urol 2019; 75: 659-66.

11. Montorsi F, Gandaglia G, Fossati N, et al. Robot-assisted salvage lymph node dissection for clinically recurrent prostate cancer. Eur Urol 2017; 72: 432-8.

12. Rischke HC, Schultze-Seemann W, Wieser G, et al. Adjuvant radiotherapy after salvage lymph node dissection because of nodal relapse of prostate cancer versus salvage lymph node dissection only. Strahlenther Onkol 2015; 191: 310-20.

13. Karnes RJ, Murphy CR, Bergstralh EJ, et al. Salvage lymph node dissection for prostate cancer nodal recurrence detected by 11C-choline positron emission tomography/computerized tomography. J Urol 2015; 193: 111-6.

14. Suardi N, Gandaglia G, Gallina A, et al. Long-term outcomes of salvage lymph node dissection for clinically recurrent prostate cancer: results of a single-institution series with a minimum follow-up of 5 years. Eur Urol 2015; 67: 299-309.

15. Abreu A, Fay C, Park D, et al. Robotic salvage retroperitoneal and pelvic lymph node dissection for 'node-only' recurrent prostate cancer: technique and initial series. BJU Int 2017; 120: 401-8.

16. Jilg CA, Schultze-Seemann W, Drendel V, et al. Detection of lymph node metastasis in patients with nodal prostate cancer relapse using 18F/11C-choline positron emission tomography/ computerized tomography. J Urol 2014; 192: 103-11.

17. Deconinck S, Tosco L, Merckx L, et al. Anatomical mapping of lymph nodes in patients receiving salvage lymphadenectomy based on a positive 11C-choline positron emission tomography/ computed tomography scan. Cent Eur J Urol 2019; 72: 232-9.

18. Winter A, Henke RP, Wawroschek F. Targeted salvage lymphadenectomy in patients treated with radical prostatectomy with biochemical recurrence: complete biochemical response without adjuvant therapy in patients with low volume lymph node recurrence over a long-term follow-up. BMC Urol 2015; 15: 10 .

19. Siriwardana A, Thompson J, van Leeuwen PJ, et al. Initial multicentre experience of 68 gallium-PSMA PET/CT guided robot-assisted salvage lymphadenectomy: acceptable safety profile but oncological benefit appears limited. BJU Int 2017; 120: 673-81.

20. Häcker A, Jeschke S, Leeb K, et al. Detection of pelvic lymph node metastases in patients with clinically localized prostate cancer: comparison of [18F]fluorocholine positron emission tomography-computerized tomography and laparoscopic radioisotope guided sentinel lymph node dissection. J Urol 2006; 176: 2014-9.

21. Kolontarev K, Govorov A, Kasyan G, et al. Extended robotic salvage lymphadenectomy in patients with 'node-only' prostate 
cancer recurrence: initial experience. Cent Eur J Urol 2018; 71: 162-7.

22. Schilling D, Schlemmer HP, Wagner PH, et al. Histological verification of 11 C-choline-positron emission/computed tomography-positive lymph nodes in patients with biochemical failure after treatment for localized prostate cancer. BJU Int 2008; 102 446-51.

23. Rybalov M, Breeuwsma AJ, Leliveld AM, et al. Impact of total PSA, PSA doubling time and PSA doubling time and PSA velocity on detection rates of 11C-Choline positron emission tomography in recurrent prostate cancer. World J Urol 2013; 31: 319-23.

24. Grivas PD, Robins DM, Hussain M. Predicting response to hormonal therapy and survival in men with hormone sensitive metastatic prostate cancer. Crit Rev Oncol Hematol 2013; 85: 82-93.

Received: 19.07.2020, accepted: 16.09.2020. 